\title{
Classification of Epileptic and Non-Epileptic Electroencephalogram (EEG) Signals Using Fractal Analysis and Support Vector Regression
}

\author{
G. Buchanna ${ }^{1 *}$, P. Premchand ${ }^{2}$, A. Govardhan ${ }^{3}$ \\ ${ }^{I}$ Research Scholar, Department of Computer Science \& Engineering, Osmania University, India. \\ ${ }^{2}$ Professor, Department of Computer Science \& Engineering, Osmania University, India. \\ ${ }^{3}$ Professor, Department of Computer Science \& Engineering, JNTUH, India.
}

\begin{abstract}
Seizures are a common symptom of this neurological condition, which is caused by the discharge of brain nerve cells at an excessively fast rate. Chaos, nonlinearity, and other nonlinearities are common features of scalp and intracranial Electroencephalogram (EEG) data recorded in clinics. EEG signals that aren't immediately evident are challenging to categories because of their complexity. The Gradient Boost Decision Tree (GBDT) classifier was used to classify the majority of the EEG signal segments automatically. According to this study, the Hurst exponent, in combination with AFA, is an efficient way to identify epileptic signals. As with any fractal analysis approach, there are problems and factors to keep in mind, such as identifying whether or not linear scaling areas are present. These signals were classified as either epileptic or non-epileptic by using a combination of GBDT and a Support Vector Regression (SVR). The combined method's identification accuracy was $98.23 \%$. This study sheds light on the effectiveness of AFA feature extraction and GBDT classifiers in EEG classification. The findings can be utilized to develop theoretical guidance for the clinical identification and prediction of epileptic EEG signals.
\end{abstract}

\section{Keywords:}

Hurst Exponent;

Adaptive Fractal Analysis; EEG;

Support Vector Regression;

Gradient Boosting Decision Trees.

\section{Article History:}

Received: $\quad 02 \quad$ September 2021

Revised: $\quad 03 \quad$ November 2021

Accepted: $07 \quad$ January 2022

Published: $01 \quad$ February 2022

\section{1- Introduction}

An epileptic condition is caused by seizures associated with altered brain activity, is a Central Nervous System (CNS) malfunction. Symptoms of these seizures include loss of consciousness, odd behaviour, and perplexity. Two of the most common ways people get injured when suffering from these symptoms are by falling or biting their tongue. The start of a seizure is difficult to anticipate before it happens. Because most seizures happen rapidly, many researchers have struggled to establish techniques for predicting when someone is likely to have a seizure. Classification algorithms, like the one employed in this research, can help predict whether or not a person will experience a seizure. Andrzejak et al., investigators have concentrated their efforts on identifying the nonlinear deterministic dynamics seen in seizures to better comprehend the brain's electrical activity and dynamic characteristics [1]. Ott et al. [2] have used a set of differential equations to explain the dynamic brain system. Electroencephalograms (E.E.G.s) can also be explained using a technique known as Nonlinear Time Series Analysis (NTSA) [3-5]. Numerous studies have looked at brain scans from people with diseases including Parkinson's [6] and depression [7], as well as those from persons in good health [9-11] and epilepsy sufferers, which is the subject of this research [12-22].

* CONTACT: drbuchannagajula@gmail.com

DOI: http://dx.doi.org/10.28991/ESJ-2022-06-01-011

(C) 2022 by the authors. Licensee ESJ, Italy. This is an open access article under the terms and conditions of the Creative Commons Attribution (CC-BY) license (https://creativecommons.org/licenses/by/4.0/). 
Researchers have obtained a new understanding of the brain's dynamic mechanism as a result of their findings. Epilepsy-related papers have generated data that may be split into two categories: epileptic seizures $[17,22]$ and brain activity of healthy participants [23]. Due to the nonlinearity of human brain behaviour, investigators were able to categorize and simulate it using these two situations as examples. Further research has revealed that even a little change in the brain's dynamic system characteristics can cause various functional activities of the brain $[24,25]$ and that this shift may be linked to the brain [26-28] or alternative issues [29,30]. Seizures associated with epilepsy are still problematic for many scientists [13,17-20]. This study's goal is to find the best classification algorithm for classifying a person is having a seizure or not. It also aims to find out how the classification algorithm responds to changes in the classification parameter settings. The technique described in Gautama et al. (2003) [31] study, enables for detailed characterization of EEG time series data, which may subsequently be utilized to enhance signal categorization. According to Nigam \& Graupe (2004) [32], a neural network-based epilepsy detection system for identifying epileptic seizures from EEG data was proposed, which was based on neural network technology. Güler \& Übeyli (2005), Subasi (2007), Adeli et al. (2007), Guo et al. (2010), and Srinivasan et al. (2005) [33-37] explored electroencephalogram signal classification based on wavelet coefficients. To accomplish the adaptive capacity, a neural network was coupled with fuzzy logic and persistent transformations of the probability density function. They looked at the E.E.G. signal subbands in terms of frequency and used multi-resolution decomposition as well as an artificial neural network on the signals. It's been proposed to utilize a special kind of recurrent neural network for automatically identifying epileptic episodes. EEG signals and real data were used to create correlation metrics that were then verified by Harikrishnan et al. (2006) and Kannathal et al. (2005) [38, 39].

Epileptic seizures were detected using various entropy estimators applied to EEG data from epileptic and normal individuals in Kannathal et al. (2005), Srinivasan et al. (2007) and Nicolaou \& Georgiou (2012) [40-42]. Übeyli \& Güler (2007) [43] presented a pattern recognition-based eigenvector feature extraction technique for EEG signal detection, and Polat \& Güneş (2008) [44] established a novel hybrid automated identification system for EEG signal categorization based on pattern recognition. Using a back-propagation neural network, Kumar et al. (2014) [45] categorized EEG data using a feed-forward technique and Subasi \& Gursoy (2010) [46] identified epileptic seizures using a back-propagation neural network with Principal Component Analysis (P.C.A.). When it comes to epilepsy treatments, one group of researchers suggested using a multilayer perceptron neural network-based categorization approach, while another group suggested using basis-based wavelet packet entropy for EEG feature extraction to improve seizure detection performance discussed in Orhan et al. (2011), Wang et al. (2017), Gajic et al. (2015), and Wang et al. (2011) [47-50]. Referencing [51] employed sophisticated artificial intelligence to detect epileptic seizures in EEG data, whereas Referencing [52] utilized sub-band nonlinear characteristics to detect seizures in EEG signals. Epilepsy detection using the DWT and KNN classifier was recently suggested [53]. Epileptic seizures can be detected using an E.E.G. signal classification approach recently proposed by Alickovic et al. (2018) [54]. Subasi et al. (2019), Hussain (2018), Rosas-Romero et al. (2019) and Gusnanto et al. (2007) [56-59] described convolutional neural networks for predicting epileptic seizures. The frequency of sub-bands that are analyzed with high frequency with several spectral thresholds [66]. The well-known ANN-BP methods have been used on datasets with distinct and substantial time-frequency domain characteristics [67]. The use of Fractional Fourier Transform (FrFT) has been proposed as a novel technique for acquiring high-resolution Synthetic Aperture Radar (SAR) pictures [68]. The autoencoder that investigates the non-linear dynamics of EEG signals [69]. 10-fold cross-validation with Bi-LSTM classifier in a supervisor deep convolutional autoencoder model for classification of EEG signals [70].

The rest of the paper is organized as Section 2 describes, with the proposed method, the experimental results, and discussions discussed in Section 3. Finally, the observations from the proposed method and future remarks are mentioned.

\section{2- Proposed Method}

This section described the proposed methodology. Figure 1 is an architecture of the classification of seizures using adaptive fractal analysis, Hurst estimation, support vector regression and multilayer gradient boosting decision trees algorithms. Here, inputting the dataset, pre-processed the data, the features extracted, then train the model using SVR and mGBDT, and the model is evaluated with test data and found that the model runs with good accuracy.

The main contribution of the paper is as follows:

- Adaptive fractal analysis (AFA) insensitive to noise and the Hurst exponent were both used as characteristics in this study.

- Support Vector Regression (SVR) improves the accuracy of signal recognition when the signal's complexity is not immediately apparent.

- The mutual information and gradient boosting decision trees approach, which does not require the sequence length of the epileptic signals, is used for the short-term epileptic signals to find the appropriate time delay and different measurement parameters. 


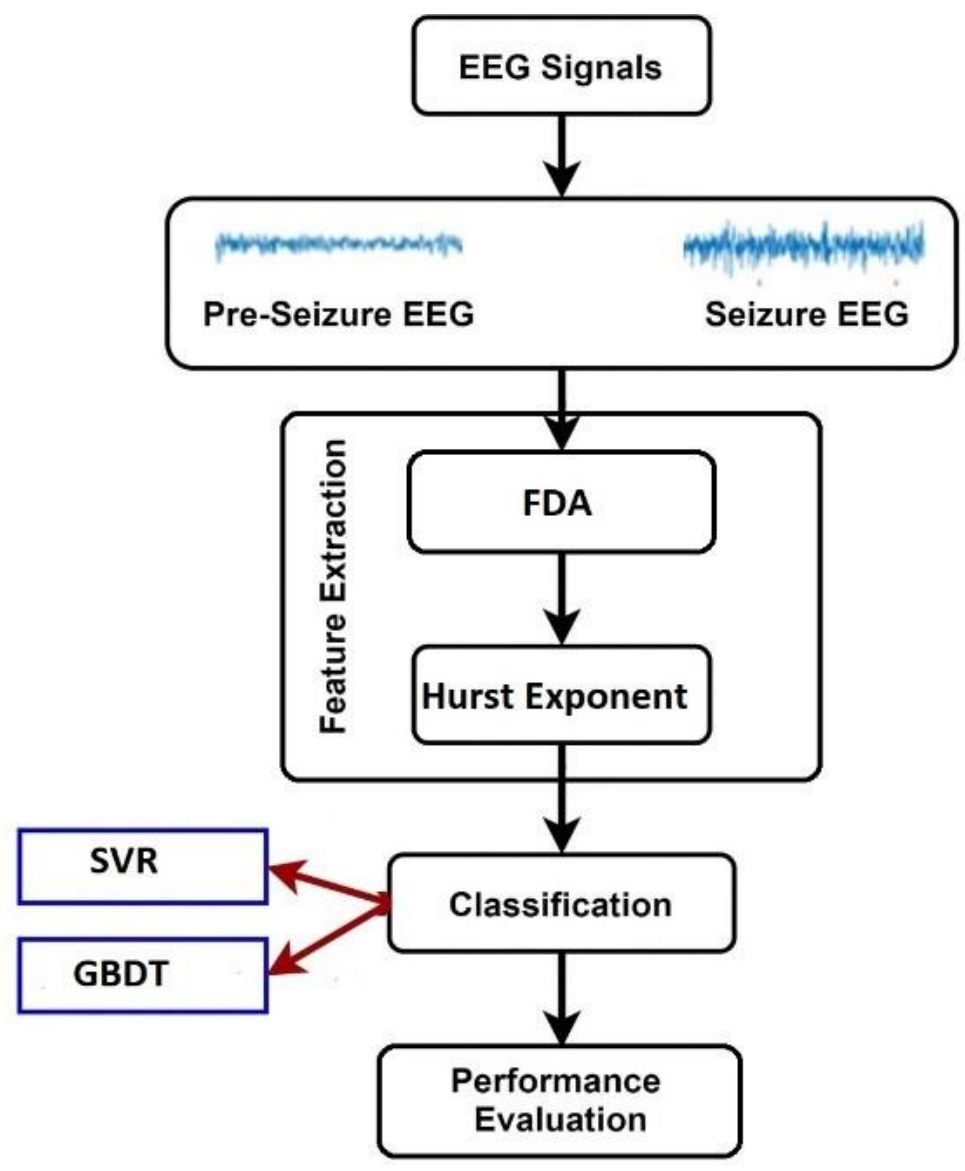

Figure 1. The Architecture of FDA and SVR

The Hurst exponent of the data set may be calculated by examining the scaling properties of the "rescaled range" of the data as follows: consider a dataset $\{\mathrm{yt}\}(\mathrm{t}=1,2,3 \ldots \mathrm{T})$, and let $(\mathrm{yi}, \mathrm{yi}+1, \ldots \mathrm{yi}+\tau), \tau<=\mathrm{T}, \mathrm{i}=1,2,3$, T- $\tau$ represents any sequence of $\tau+1$ points within the data set. The rescaled range $(\mathrm{R} / \mathrm{S})$ statistic is then defined as:

$$
\left(\frac{\mathrm{R}}{\mathrm{s}}\right)_{\tau}=\frac{1}{\mathrm{~s}_{\tau}}\left[\sup \sum_{\mathrm{k}=\mathrm{i}}^{\mathrm{t}}\left(\mathrm{x}_{\mathrm{k}}-\mathrm{x}_{\mathrm{i}, \tau}\right)-\inf \sum_{\mathrm{k}=1}^{\mathrm{t}}\left(\mathrm{x}_{\mathrm{k}}-\mathrm{x}_{\mathrm{i}, \tau}\right)\right]
$$

where;

$$
x_{i, \tau}=(1 / \tau) \sum_{t=1}^{\tau} x_{t}
$$

Equation 2 is the sample mean and Equation 3 is SD:

$S_{i, \tau}=\left[\frac{1}{\tau} \sum_{t=i}^{\tau}\left(x_{t}-x_{i, \tau}\right)^{2}\right]^{1 / 2}$

$\left\langle\left(\frac{\mathrm{R}}{\mathrm{S}}\right)_{\tau}\right\rangle_{\mathrm{i}}$

The quantity, Equation 4 is proportional to $\tau^{\mathrm{H}}$, such that the gradient of a plot of $\left(\left\langle(R / S)_{\tau}\right\rangle_{i}\right)$ vs $\log (\tau)$ equals to $\mathrm{H}$. Also, the Hurst exponent can be measured as a function of interval width. A typical analysis employs the "variance method" to determine the various features within the time interval, as mentioned in Equation 5:

$$
\mathrm{V}(\Delta \mathrm{t})=\left\langle\left[\mathrm{y}_{\mathrm{t}+\Delta \mathrm{t}}-\mathrm{y}_{\mathrm{t}}\right]^{2}\right\rangle_{\mathrm{t}}
$$

$\Delta \mathrm{t}$ is the range of values, this amount is connected to the Hurst exponent as $\mathrm{V}(\Delta \mathrm{t}) \propto \Delta \mathrm{t}_{2} \mathrm{H}$, hence the range of $\Delta \mathrm{t}$ values against variance of these $\Delta t$ values can be observed as linear values, but the estimation of variance will be poor. For each point ti in the trace, the min and max values of xt0 learned as upper (u) and lower (b) correspondingly for an oscillation, that reflects $\mathrm{u}_{\epsilon}\left(\mathrm{t}_{\mathrm{i}}\right)$ and $\mathrm{b}_{\epsilon}\left(\mathrm{t}_{\mathrm{i}}\right)$ the upper and lower oscillation wrappers at a specified range of width. After the estimation of the Hurst exponent, apply adaptive fractal analysis [10] for extracting time-series fractal features. Dubuc's variation method and AFA both look at the geometric properties of a time series trace. The time-series chunks are built using AFA at various approximations and are recorded with a variance of the accuracy of the oscillation. 


\section{2-1-The AFA Algorithm}

AFA extracts globally smooth trend signals from the data using an adaptive fractal analysis method then examine the scaling of the residuals to the fit as a function of the time scale at which the fit is produced. Assume $y(t)=(t=1,2,3 . T)$ Select a window $\mathrm{w}=2 \mathrm{n}+1, \mathrm{w}<\mathrm{T}$ and divide the data set into overlapping subsets of length $\mathrm{w}$ that overlap by $\mathrm{n} 1$ data points. The best-fitting polynomial of order $\mathrm{M}$ is found within each window. This is accomplished using normal leastsquares regression, which involves adjusting the coefficients of the polynomial model until it best fits the data. Although raising the order $\mathrm{M}$ can typically improve the fit accuracy, it is important to avoid over-fitting the sample. $\mathrm{M}$ is usually 1 or 2, indicating a straight or exponential function. The objective isn't to fit the polynomial framework to every zigzag or fluctuation in $\mathrm{u}(\mathrm{i})$, but rather to capture any generally broad trends in the data while allowing enough leftover variation to investigate further.

To calculate the linear best-fit line for each window, a series of disconnected straight lines is generated. That is, each index in the original data set's domain corresponds to a point on each of two subset fit lines (except for the $n$ data points at either end of the trace). Next, these best-fit lines are "stitched" together to form a single, smoothly continuous curve in the following manner. Integers from 1 to $(n+1)$ should be used to identify the windows that span the trace, and $y(j)(1)$ should be used to identify the best-fitting lines. To create the curve in each of the $\mathrm{j}$ windows, use Equation 6 :

$$
\mathrm{y}^{(\mathrm{w})}(\mathrm{l})=\mathrm{w}_{1} \mathrm{y}^{(\mathrm{j})}(\mathrm{l}+\mathrm{n})+\mathrm{w}_{2} \mathrm{y}^{(\mathrm{j}+1)}(\mathrm{l}),
$$

where $\mathrm{w} 1=(1-(1-1) / \mathrm{n})$ and $\mathrm{w} 2=(1-1) / \mathrm{n}$.

The index at each $y(w)$ (l) value is the weighted average of the two best-fit lines, which equals the distance between the index and the window's midpoint. The trace $\mathrm{y}(\mathrm{w})(\mathrm{t})$ that results from doing this method across all windows approximates the trace $\mathrm{x}(\mathrm{t})$ on the length scale given by $\mathrm{w}$. The Hurst exponent is determined by the scaling behaviour of this fidelity as $w$ is varied, especially;

$$
F(w)=\left[\frac{1}{T} \sum_{i=1}^{\mathrm{T}} \mathrm{y}^{\mathrm{w}}\left(\mathrm{t}_{\mathrm{i}}\right)-\mathrm{x}\left(\mathrm{t}_{\mathrm{i}}\right)^{2}\right]^{1 / 2} \alpha \mathrm{w}^{\mathrm{H}}
$$

The graph of $\mathrm{F}(\mathrm{w})$ against $\log (\mathrm{w})$ has a slope of $\mathrm{H}$, which is an important finding. The estimation of these functions is as follows:

The dataset $\mathrm{B}(\mathrm{t}, \mathrm{w})$ is thus a function whose increments $\mathrm{B}(\mathrm{t} 2, \mathrm{w})-\mathrm{B}(\mathrm{t} 1, \mathrm{w})$ have a mean of zero and variance of $\mid \mathrm{t} 2$ $\mathrm{t} 1$, and whose non-overlapping increments $\mathrm{B}(\mathrm{t} 2, \mathrm{w})-\mathrm{B}(\mathrm{t} 1, \mathrm{w})$ and $\mathrm{B}(\mathrm{t} 4, \mathrm{w})-\mathrm{B}(\mathrm{t} 3, \mathrm{w})$ are statistically independent. The equations 8, 9 are indicating a "reduced fractional Brownian motion" $\mathrm{BH}(\mathrm{t}, \mathrm{w})$, then is further characterized by the parameter $\mathrm{H}, 0<\mathrm{H}<1$ and satisfies $\mathrm{BH}(0, \mathrm{w})=\mathrm{b} 0$,

$$
\begin{aligned}
& \mathrm{B}_{\mathrm{H}}(\mathrm{t}, \mathrm{w})-\mathrm{B}_{\mathrm{H}}(0, w)=\mathrm{B}_{\mathrm{H}}(0, w)=\mathrm{b}_{0} \\
& \mathrm{~B}_{\mathrm{H}}(\mathrm{t}, \mathrm{w})-\mathrm{B}_{\mathrm{H}}(0, w)=\frac{1}{\Delta\left(\mathrm{H}+\frac{1}{2}\right)}\left\{\int_{-\infty}^{0}\left[(\mathrm{t}-\mathrm{s})^{\mathrm{H}-1 / 2}-(-\mathrm{s})^{\mathrm{H}-1 / 2}\right] d B(\mathrm{~s}, \mathrm{w})+\int_{0}^{\mathrm{t}}(\mathrm{t}-\mathrm{s})^{\mathrm{H}-\frac{1}{2}} d B(s w)\right\}
\end{aligned}
$$

As a result, equation 10 is a fractional Brownian motion trace is self-affine

$$
\left\{\mathrm{B}_{\mathrm{H}}\left(\mathrm{t}_{0}+\tau, \mathrm{w}\right)-\mathrm{B}_{\mathrm{H}}\left(\mathrm{t}_{0}, \mathrm{w}\right)\right\} \equiv\left\{\mathrm{h}^{-\mathrm{H}}\left[\mathrm{B}_{\mathrm{H}}\left(\mathrm{t}_{0}+\mathrm{h} \tau, \mathrm{w}\right)-\mathrm{B}_{\mathrm{H}}\left(\mathrm{t}_{0}, \mathrm{w}\right)\right]\right\}
$$

where;

$$
\{\mathrm{X}(\mathrm{t}, \mathrm{w})\} \equiv\{\mathrm{Y}(\mathrm{t}, \mathrm{w})\}
$$

$\mathrm{X}(\mathrm{t}, \mathrm{w})$ and $\mathrm{Y}(\mathrm{t}, \mathrm{w})$ have identical finite joint distribution functions [11]. For every additional $\mathrm{h}$, the difference between $\mathrm{BH}(\mathrm{t} 0+\mathrm{h}, \mathrm{w})$ and the $\mathrm{BH}(\mathrm{t} 0, \mathrm{w})$ rises by one. When the $\mathrm{x}$ and $\mathrm{y}$ axes are scaled independently, the statistical characteristics seen inside the intervals are maintained (specifically, $\mathrm{h}$ and $\mathrm{hH}$, respectively).

\section{2-2-AdaBoost}

AdaBoost fits a sequence of weak learners to the data using the information in the data. The algorithm subsequently gives more weight to wrong predictions while giving less weight to correct predictions. As a result, the algorithm concentrates its efforts on observations that are more difficult to anticipate. In classification, the final result is determined by the majority vote, while in regression, the final result is determined by the average. Scikit-learn can be used to create an implementation of this algorithm. It can be provided with the $\mathrm{n}$ estimators argument, which indicates the number of weak learners that are required. The learning rate option can be used to adjust the amount of contribution made by each weak learner. By default, decision trees are used as the base estimators in the algorithm, however, this can be changed. The performance of the model can be improved by fine-tuning the base estimators and the parameters of the decision trees, among other things. AdaBoost's decision trees have a single split by default, which is the most common. 
To apply the AdaBoost model for classification issues, you can make use of the Scikit-learn module AdaBoostClassifier. As you can see in the table below, the settings of the base estimator can be customized to meet your needs. In addition, the classifier accepts the number of estimators that you specify. This is the total number of decision trees that will be required for your model.

Step 1: Assume that $\mathrm{F}_{\mathrm{i}}^{\mathrm{t}-1}$ obtained from the $\mathrm{t}-1$ iteration where $\mathrm{t}$ is iteration. We need to pair $\mathrm{G}_{\mathrm{i}}^{\mathrm{t}}$ and $\mathrm{F}_{\mathrm{i}}^{\mathrm{t}-1}$, to obtain pseudo-inverse such that $\mathrm{G}_{\mathrm{i}}^{\mathrm{t}}\left(\mathrm{F}_{\mathrm{i}}^{\mathrm{t}-1}\left(\mathrm{o}_{\mathrm{i}-1}\right)\right) \cong \mathrm{o}_{\mathrm{i}-1}$. The reconstruction loss is calculated as $G_{i}^{t}=\operatorname{argmin}_{G_{i}^{t}}$ $E_{x}\left[L^{\text {inverse }} o_{i}, G_{i}^{t}\left(F_{i}^{t-1}\left(o_{i-1}\right)\right)\right]$, where $\mathrm{L}^{\text {inverse }}$ is reconstruction loss which is intended to minimize. One of the good practices is setting $\| G_{i}\left(F_{i}\left(o_{i-1}+\epsilon\right)\right)-\left(0_{i-1}+\epsilon \|, \epsilon \sim \mathcal{N}\left(0, \operatorname{diag}\left(\sigma^{2}\right)\right.\right.$, by setting like the model becomes more robust. And also inverse mapping forced to learn whom to train with neighboring training data in the right manner.

Step 2: After updating $\mathrm{G}_{\mathrm{i}}^{\mathrm{t}}$, it can be used to update $\mathrm{F}_{\mathrm{i}-1}$ layer forward mapping. The Pseudo labels are defined $\mathrm{z}_{\mathrm{i}-1}^{\mathrm{t}}=$ $G_{i}\left(z_{i}^{t}\right)$, for all intermediate layers for $t$ iteration, the pseudo labels are propagated from intermediate layers to output layers. For each layer gradient ascent step towards the pseudo-residuals as:

- $\frac{\partial L\left(F_{i}^{t-1}\left(o_{i-1}, z_{i}^{t}\right)\right.}{\partial F_{i}^{t-1}\left(o_{i}-1\right)}$ as typical regression of gradient boosting decision trees (GBDTs). The final layer pseudo-labels are update $z_{M}^{t}$ at layer M. It is easy to define pseudo-labels of the output layer as: $z_{M}^{t}=o_{M}-\alpha \frac{\delta\left(L\left(o_{M}, y\right)\right.}{\delta o_{M}}$. Finally, $F_{M}^{t}$ is fit to pseudo-residuals $-\frac{\partial \mathrm{L}\left(\mathrm{F}_{\mathrm{M}}^{\mathrm{t}-1}\left(\mathrm{o}_{\mathrm{M}-1}, \mathrm{z}_{\mathrm{M}}^{\mathrm{t}}\right)\right.}{\partial \mathrm{F}_{\mathrm{M}}^{\mathrm{t}}\left(\mathrm{o}_{\mathrm{i}}-1\right)}$. Once $\mathrm{F}_{\mathrm{i}}$ gets updated, as per procedure it will go to the next iteration to update Gi. Initializing a neural network involves assigning random Gaussian noise to each parameter, followed by parameter updating. Drawing a random tree structure from the provided tree-structured model is not easy. Figure 2 describes the process of multilayered gradient boosting decision trees.

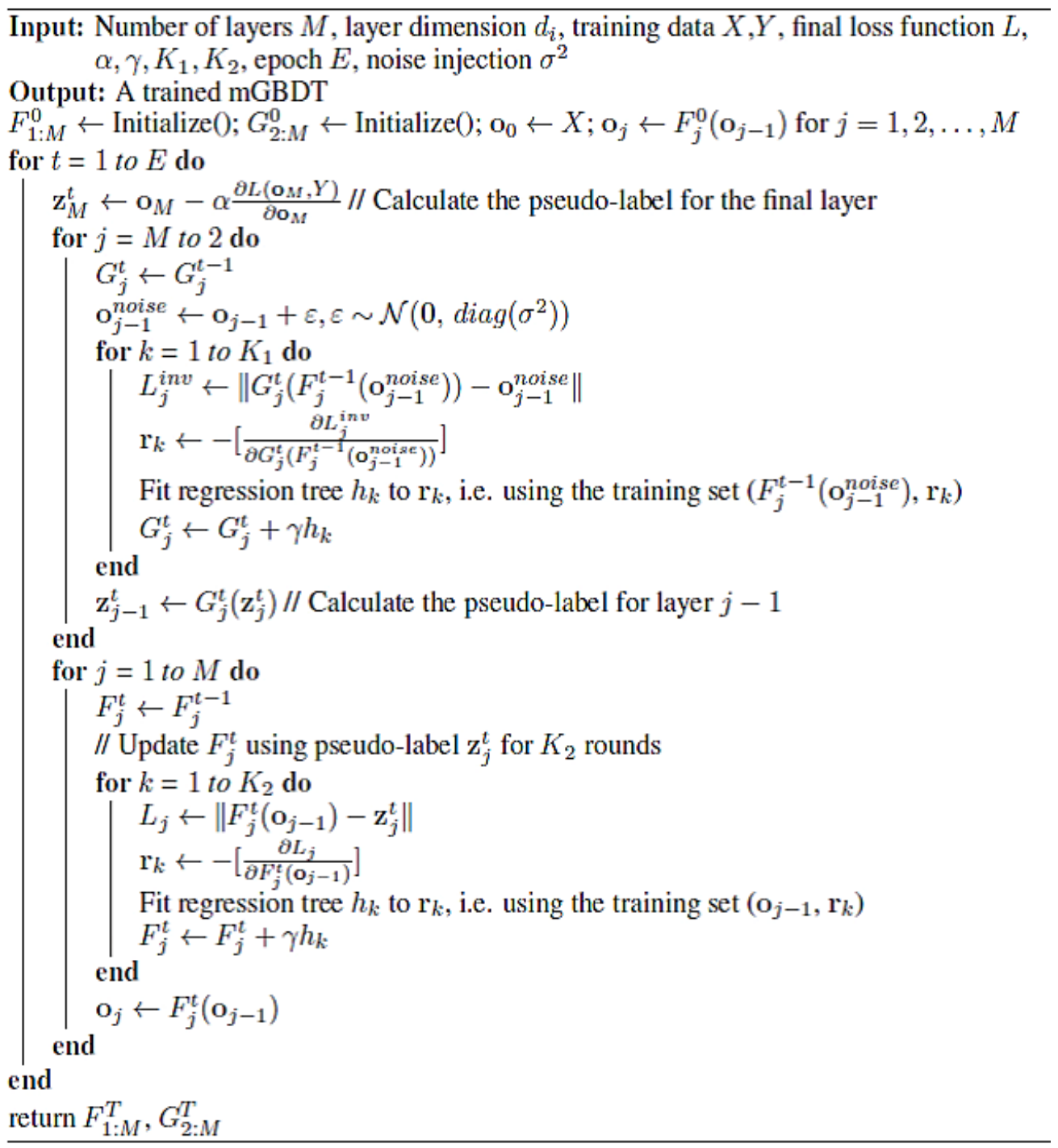




\section{2-3- Support Vector Regression (SVR)}

The training dataset $\{(\mathrm{x} 1, \mathrm{y} 1, \ldots \mathrm{xn}), \mathrm{yn}\}$ where $\mathrm{xi} \in \mathrm{Rn}$ represents $\mathrm{EEG}$ signals samples and yi are target values for $\mathrm{i}=1,2, \ldots, \mathrm{N}$. The generic SVR estimation is calculated with Equation 12:

$\mathrm{f}(\mathrm{x})=(\mathrm{w} \cdot \theta(\mathrm{x}))+\mathrm{b}$

where $x \in R n, b \in R$, and $\Theta$ represents a nonlinear transformation of high dimension. By minimizing risk, $w$ and $b$ values are calculated in Equation 13:

$$
\mathrm{R}_{\text {reg }}=\mathrm{C} \sum_{\mathrm{i}=0}^{\mathrm{n}} \pi\left(\mathrm{f}\left(\mathrm{x}_{\mathrm{i}}\right)-\mathrm{y}_{\mathrm{i}}\right)+\frac{1}{2} \cdot\left\|\mathrm{w}^{2}\right\|
$$

where $\mathrm{c}$ is constant, $\pi$ is cost function and vector ' $\mathrm{w}$ ' can rewritten as in Equation14:

$$
\mathrm{w}=\sum_{\mathrm{i}=0}^{\mathrm{n}}\left(\alpha_{\mathrm{i}}-\alpha_{\mathrm{i}}^{*}\right) \varphi(\mathrm{x})
$$

The generic equation is:

$$
f(x)=\sum_{i=0}^{n}\left(\alpha_{i}-\alpha_{i}^{*}\right) \cdot\left(\varphi\left(x_{i}\right) \cdot \varphi(x)\right)+b=\sum_{i=0}^{n}\left(\alpha_{i}-\alpha_{i}^{*}\right) \cdot k\left(x_{i}, x\right)+b
$$

where $\mathrm{k}(\mathrm{xi}, \mathrm{x})$ is the kernel function.

\section{3- Results and Discussions}

The epileptic seizure (E.S.) dataset was utilized in this study. The dataset has 11,500 samples with 178 characteristics each and is properly distributed. Where the samples are divided into five groups $\mathrm{Y}=1,2,3,4,5$; such as a) Class 5 eyes open during recoding EEG signal (E.Y.E.O. in this research) b) Class 4 - eyelids closed during recording EEG signal (E.Y.E.C. in this research). c) Class 3 - Yes, an EEG from the healthy brain area revealed a brain tumour, labelled H.S.T.U. M.O.R. d) A Class 2 EEG signal was detected near the brain tumour, labelled TUMOR. e) Class 1: Seizure recording, E.S. The method applied with four examples analyzed and were divided into two, three, and five EEG signal types.

The dataset is assessed and evaluated on the Google Collaborator notebook using Python Programming Language. Data sets are divided into two groups (no seizures and convulsions), three classes (normal, interictal and ictal), and five classes, to explore the basic categorization model (A, B, C, D, and E). We evaluated eight models of different configurations to pick optimal model parameters.

\section{3-1- Performance Measures}

The efficiency measures evaluated include Sensitivity (SEN), specificity (SPE), absolute accuracy (TA), kappa value (KV), and random accuracy (RA) [66]. Many of these measurements are expressed as true-positive (TP), false-positive (FP), true-negative (TN), and false-negative (FN) defined in Equations 16 to 20:

$$
\begin{aligned}
& \mathrm{SEN}=\frac{\mathrm{TP}}{\mathrm{TP}+\mathrm{FN}} \\
& \mathrm{SPE}=\frac{\mathrm{TN}}{\mathrm{TN}+\mathrm{FP}} \\
& \mathrm{TA}=\frac{\mathrm{TP}+\mathrm{TN}}{\mathrm{TP}+\mathrm{FP}+\mathrm{TN}+\mathrm{FN}} \\
& \mathrm{RA}=\frac{(\mathrm{TN}+\mathrm{FP}) *(\mathrm{TN}+\mathrm{FN})+(\mathrm{FN}+\mathrm{TP}) *(\mathrm{FP}+\mathrm{TP})}{(\mathrm{TP}+\mathrm{FP}+\mathrm{TN}+\mathrm{FN})^{2}} \\
& \mathrm{k}=\frac{\mathrm{TA}-\mathrm{RA}}{1-\mathrm{RA}}
\end{aligned}
$$

Various statistical tests based on class distances have been presented [59, 60]. Among them, the area under the curve (AUC) and the partial area at pre-determined great specificity inception (pAUC) of ROC curves were used [61, 62]. A ROC curve depicts the relationship between the TPF and FPF from a series of twofold classification experiments. The TPF indicates sensitivity, while FPF denotes specificity. In the case of a gene expression profile, AUC calculates the chance that a subject randomly picked from one class (e.g., a group of people suffering from a specific disease) will have a greater expression value than another class (e.g., healthy people) [62].

Figure 3 shows the AUC curve performance of the proposed strategy. From the plot, it is observed that the AUC for the Z-S ROC curve is significantly greater than the AUC for the ZO-FN-S and Z-O-F-N-S ROC curves. As a result, we may conclude that logistic regression performed significantly better than binary classification in categorizing the positive class in the dataset. 


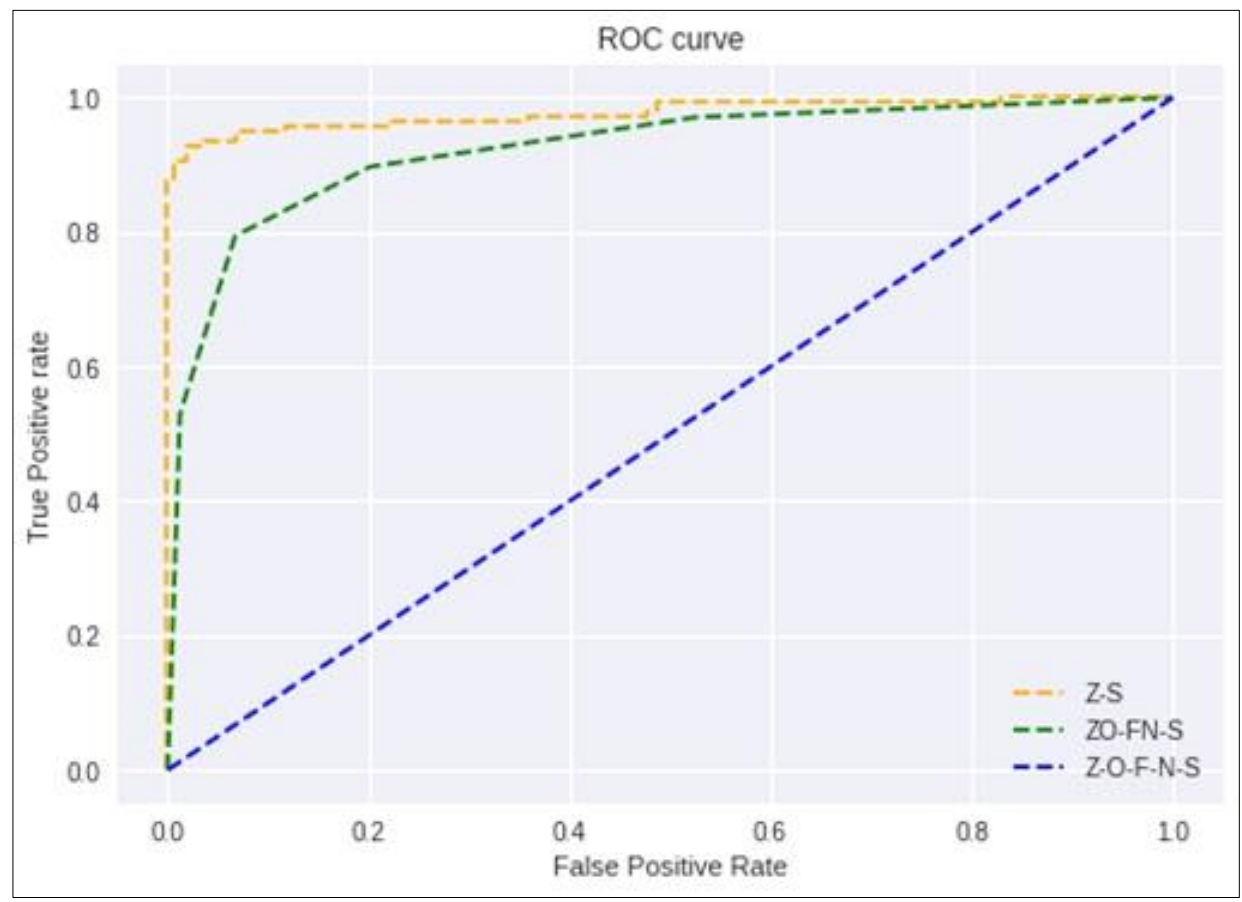

Figure 3. AUC Curve of three classes

The AUC-ROC curve is for binary classification. The One vs. All strategy can be used to solve multiclass classification problems. Z-S, ZO-FN-S, and Z-O-F-N-S will be classified as 0 against 1 and 2, respectively. Classifying 1 versus not 1 generates the ROC for class 1 . The multi-class classification ROC curve is as follows (Figure 4):

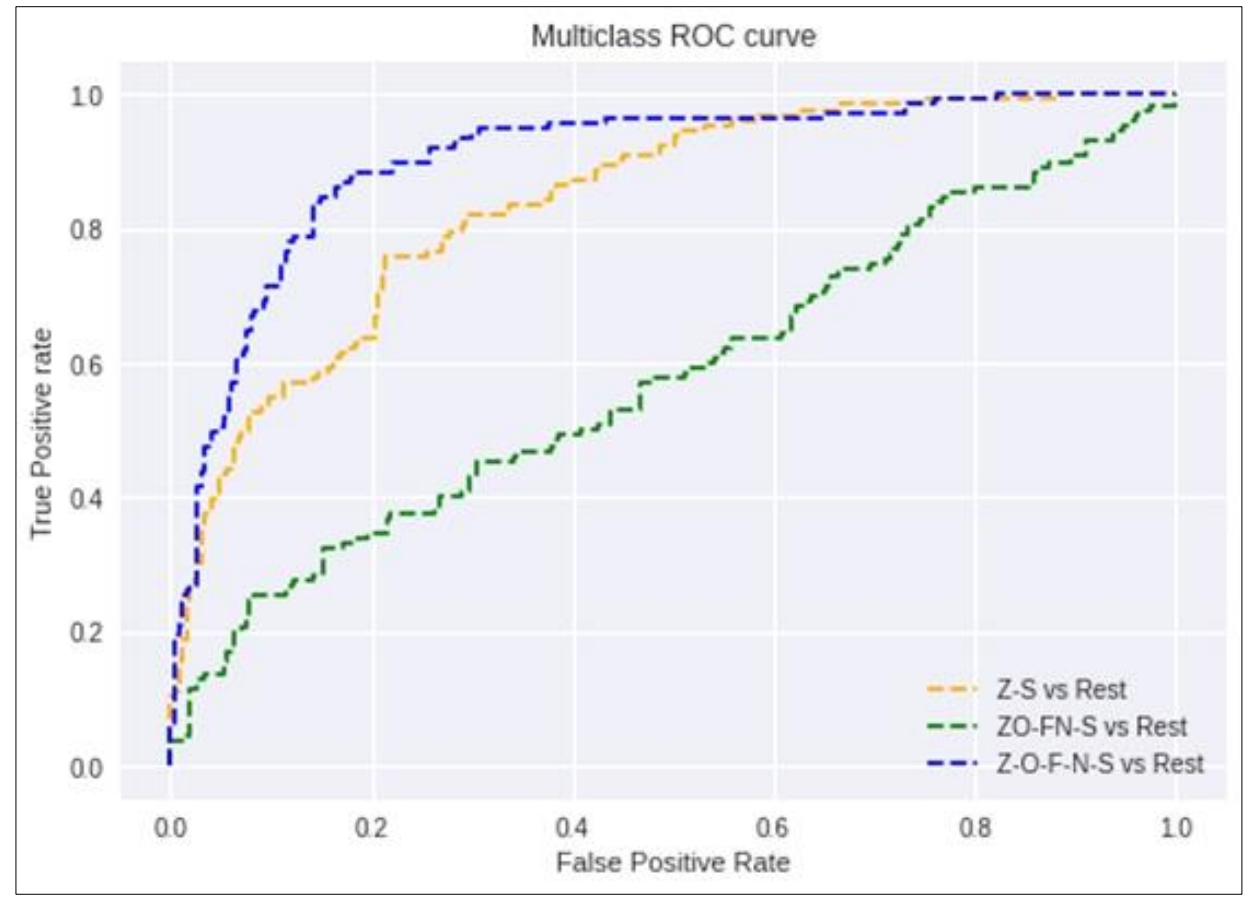

Figure 4. Multi-class ROC curve of the proposed strategy

Figure 4 shows, ROC curve of the proposed method for multi-class classification (Considered three classes). When it comes to statistics, the mean absolute error (MAE) is a measure of the differences in errors between paired observations that describe the same event. For example, comparisons of expected against observed values, subsequent time against starting time, and one measuring technique versus an alternate measurement technique are all examples of $\mathrm{Y}$ versus $\mathrm{X}$. The MAE is determined as follows:

$$
\text { MAE }=\frac{\sum_{\mathrm{i}=1}^{\mathrm{n}}\left|\mathrm{y}_{\mathrm{i}}-\mathrm{x}_{\mathrm{i}}\right|}{\mathrm{n}}=\frac{\sum_{\mathrm{i}=1}^{\mathrm{n}}\left|\mathrm{e}_{\mathrm{i}}\right|}{\mathrm{n}}
$$

The absolute error $\left|e_{i}\right|=\mid y_{i}-x_{i}$, and $y_{i}$ is prediction and $x_{i}$ is true value. The Root Mean Square Deviation is a measurement of quality to fit the model using regression analysis and is given as follows: 


$$
\operatorname{RMSE}=\sqrt{\sum_{\mathrm{i}=1}^{\mathrm{n}}\left(\mathrm{y}_{\mathrm{i}}-\mathrm{y}\right)^{2}}
$$

$\mathrm{Y}_{\mathrm{i}}$ is $\mathrm{i}^{\text {th }}$ observation and $\mathrm{y}$ is the predicted value. Tables 1 and 2, show the findings of the proposed method in terms of TA, SEN, SPE, RA, K, Accuracy, ROC, MAE, RMSE and Time. Each factor value shows the superiority of the proposed method.

Table 1. TA, SEN, SPE value of three classes of proposed method

\begin{tabular}{cccccc}
\hline Classes & $\begin{array}{c}\text { TA } \\
(\boldsymbol{\%})\end{array}$ & $\begin{array}{c}\text { SEN } \\
(\boldsymbol{\%})\end{array}$ & $\begin{array}{c}\text { SPE } \\
(\boldsymbol{\%})\end{array}$ & RA & K \\
\hline Z-S & 99.33 & 99.08 & 98.75 & 0.788 & 99.33 \\
ZO-FN-S & 98.53 & 97.45 & 97.75 & 0.675 & 98.53 \\
Z-O-F-N-S & 97.42 & 96.75 & 95.84 & 0.546 & 97.42 \\
\hline
\end{tabular}

Table 2. Measurement values of the proposed method of three classes

\begin{tabular}{cccccc}
\hline Classes & Accuracy & ROC & MAE & RMSE & Time \\
\hline Z-S & 98.23 & 0.587 & 0.1823 & 0.4532 & 4.51 \\
ZO-FN-S & 97.52 & 0.568 & 0.1821 & 0.4231 & 4.35 \\
Z-O-F-N-S & 96.32 & 0.523 & 0.1811 & 0.4111 & 4.23 \\
\hline
\end{tabular}

The above table clearly states that Z-S class (binary classification given high accuracy i.e. 98.23 when compare with ZO-FN-S and Z-O-F-N-S, the respective ROC, MAE, RMSE and Time are also shown. The experiment is conducted for different Epochs i.e. 500, 1000 and 1500 and respective results with ROC, MAE, and RMSE measures are shown in Table 3. The output layer has three labels like Z-S, ZO-FN-S and Z-O-F-N-S. For each label or class, concerning 500, 1000, 1500 epochs the accuracy value is mentioned in Table 3.

Table 3. Results of proposed method for different epochs

\begin{tabular}{ccccccc}
\hline Classes & Epoch & Accuracy & ROC & MAE & RMSE & Time \\
\hline \multirow{2}{*}{ Z-S } & 500 & 95.62 & 0.456 & 0.1894 & 0.3599 & 3.56 \\
& 1000 & 97.23 & 0.458 & 0.1852 & 0.3598 & 4.11 \\
& 1500 & 98.23 & 0.587 & 0.1752 & 0.3561 & 4.51 \\
\hline \multirow{2}{*}{ ZO-FN-S } & 500 & 97.52 & 0.568 & 0.1821 & 0.4231 & 4.35 \\
& 1000 & 97.85 & 0.568 & 0.1812 & 0.4228 & 4.69 \\
& 1500 & 97.89 & 0.586 & 0.1811 & 0.4211 & 5.21 \\
\hline \multirow{2}{*}{ Z-O-F-N-S } & 1000 & 96.85 & 0.532 & 0.1852 & 0.4211 & 4.68 \\
& 1500 & 96.94 & 0.541 & 0.1855 & 0.4352 & 4.98 \\
\hline
\end{tabular}

\section{3-2-Comparative Analysis}

The results of the proposed method were compared with other methods in the literature. In our study, the experimental results were compared with their classification accuracy rates and statistical analysis results. Hence, the proposed methods listed in Table 4 were used to test their performances for classifying $\{Z, O, N, F, S\}$ or $\{A, B, C, D, E\}$ signals.

Table 4. Comparison of proposed method results with other methods

\begin{tabular}{cccc}
\hline Methods & Methods and features & Classes & Accuracy (\%) \\
\hline \multirow{2}{*}{ Patidar and Panigrahi [63] } & TQWT & Z-S & 97.75 \\
& ZO-FN-S & 96.35 & 95.62 \\
\hline \multirow{2}{*}{ Acharya et al. [64] } & Z-O-F-N-S & Z-S & 88.70 \\
& Z layers deep CNN & ZO-FN-S & 86.32 \\
Zhou et al. [65] & Z-layers CNN & Z-O-F-N-S & 95.32 \\
\hline & & ZO-FN-S & 92.35 \\
Proposed model & Hurst exponent + adaptive fractal analysis (AFA) + GBDT & ZO-FN-S & 97.52 \\
\hline
\end{tabular}


Table 4 shows, the comparison of the proposed method with other methods and it's clear that binary classification namely Z-S has good accuracy i.e. 98.23 than multiclass classification. Figure 5 explains the accuracy of each model from the comparative methods.

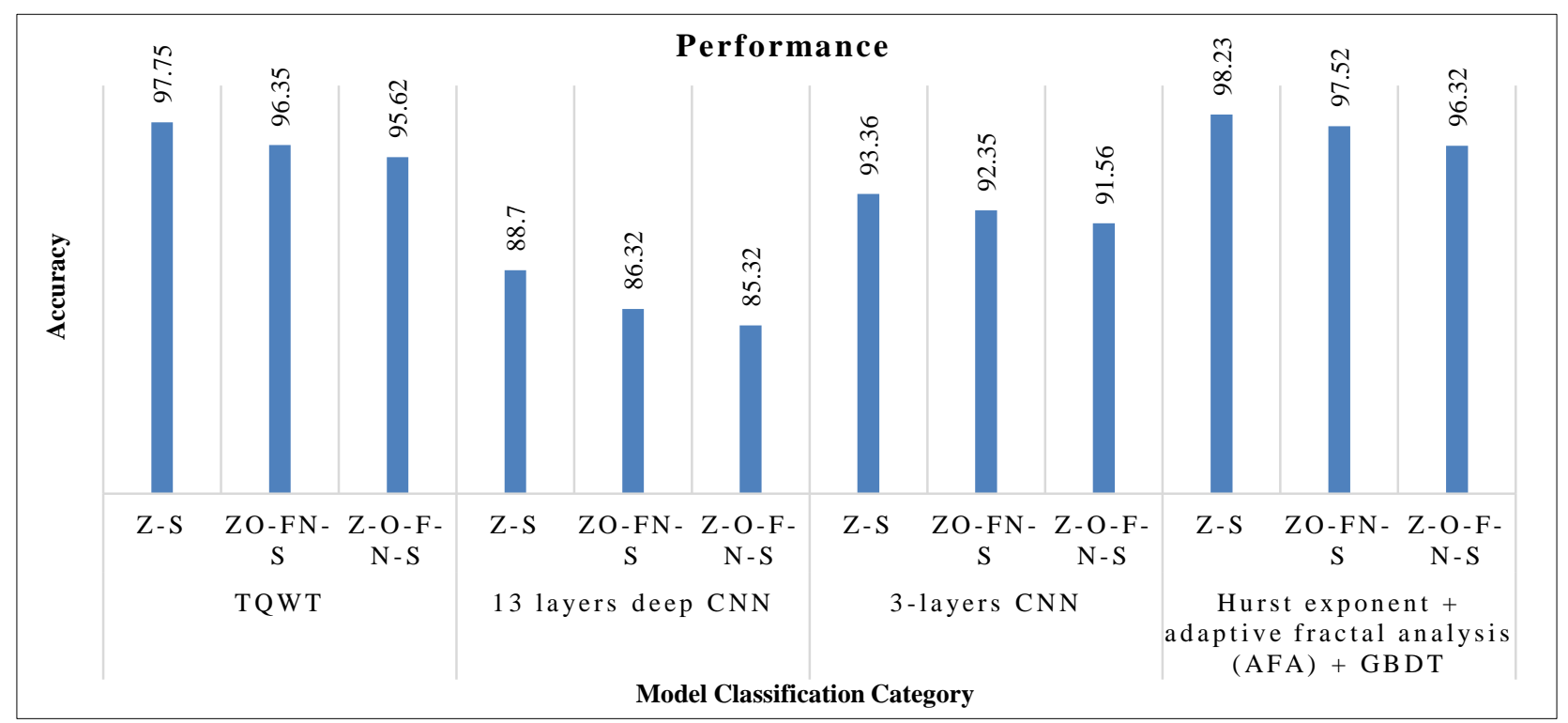

Figure 5. The accuracy of existing and proposed models

\section{4- Conclusion}

An abrupt electrical disruption is a seizure in the brain that is uncontrolled. There are two categories of convulsive and non-convulsive seizures, based on their intensity. With an abnormal EEG, we imply that the patient's epileptic condition is not convulsive. An epileptic seizure is classified in terms of three classes: Z-S, ZO-FN-S, and Z-O-F-N-S.

In this paper, an adaptive fractal analysis method was used to extract the features. Fractal analysis is a modern approach to analyzing patterns that transcend conventional Euclidean comprehension through the use of unconventional mathematical ideas. The fractal dimension is used to gauge the level of complexity. The Hurst exponent is a measure of a time series' long-term memory. The fractal dimension, $\mathrm{D}$, is closely connected to $\mathrm{H}$, which is a measure of a data series' "mild" or "wild" unpredictability. When referring to the Hurst exponent, you may hear the terms "index of dependency" or "index of long-range dependence". Support Vector Regression (SVR) with Gradient Boost Decision Tree (GBDT) is used to classify non-epileptic EEG data with high accuracy. Different measurement variables including ROC, AUC, TA, SEN, SPE, RA and K were computed for the suggested technique in this article. In all cases, epileptic and nonepileptic EEG signals were classified more accurately using the suggested approach. Three classes of EEG datasets have been used to test the suggested technique and the accuracy values are 98.23, 97.52, and 96.32\% correspondingly. Compared to the Patidar and Panigrahi [63], who achieved an accuracy of about 97.75\%, Acharya et al. [64] obtained an accuracy of $88.70 \%$, and Zhou et al. [65] reached an accuracy of $93.36 \%$. Finally, the performance of the models has yet again been confirmed by employing the classifier with some photos never seen by the systems.

\section{5- Declarations}

\section{5-1-Author Contributions}

Conceptualization, G.B.; methodology, G.B., P.P., and A.G.; software, G.B.; validation, G.B.; writing —original draft preparation, G.B.; writing - review and editing, P.P., and A.G. All authors have read and agreed to the published version of the manuscript.

\section{5-2-Data Availability Statement}

The data presented in this study are available in the article.

\section{5-3-Funding}

The authors received no financial support for the research, authorship, and/or publication of this article.

\section{5-4- Conflicts of Interest}

The authors declare that there is no conflict of interest regarding the publication of this manuscript. In addition, the ethical issues, including plagiarism, informed consent, misconduct, data fabrication and/or falsification, double publication and/or submission, and redundancies have been completely observed by the authors. 


\section{6- References}

[1] Andrzejak, R. G., Lehnertz, K., Mormann, F., Rieke, C., David, P., \& Elger, C. E. (2001). Indications of nonlinear deterministic and finite-dimensional structures in time series of brain electrical activity: Dependence on recording region and brain state. Physical Review E, 64(6). doi:10.1103/physreve.64.061907.

[2] Ott, E., Sauer, T., \& Yorke, J. A. (1994). Coping with chaos. Wiley.

[3] Rombouts, S. A. R. B., Keunen, R. W. M., \& Stam, C. J. (1995). Investigation of nonlinear structure in multichannel EEG. Physics Letters A, 202(5-6), 352-358. doi:10.1016/0375-9601(95)00335-Z.

[4] Lopes Da Silva, F. H., Pijn, J. P., Velis, D., \& Nijssen, P. C. G. (1997). Alpha rhythms: Noise, dynamics and models. International Journal of Psychophysiology, 26(1-3), 237-249. doi:10.1016/S0167-8760(97)00767-8.

[5] Pritchard, W. S., Duke, D. W., \& Krieble, K. K. (1995). Dimensional analysis of resting human EEG II: Surrogate-data testing indicates nonlinearity but not low-dimensional chaos. Psychophysiology, 32(5), 486-491. doi:10.1111/j.14698986.1995.tb02100.x.

[6] Pezard, L., Jech, R., \& Růžička, E. (2001). Investigation of non-linear properties of multichannel EEG in the early stages of Parkinson's disease. Clinical Neurophysiology, 112(1), 38-45. doi:10.1016/S1388-2457(00)00512-5.

[7] Nandrino, J. L., Pezard, L., Martinerie, J., El Massioui, F., Renault, B., Jouvent, R., Allilaire, J. F., \& Widlöcher, D. (1994). Decrease of complexity in EEG as a symptom of depression. NeuroReport, 5(4), 528-530. doi:10.1097/00001756-19940112000042

[8] Jeong, J., Chae, J. H., Kim, S. Y., \& Han, S. H. (2001). Nonlinear dynamic analysis of the EEG in patients with Alzheimer's disease and vascular dementia. Journal of Clinical Neurophysiology, 18(1), 58-67. doi:10.1097/00004691-200101000-00010.

[9] Soong, A. C. K., \& Stuart, C. I. J. M. (1989). Evidence of chaotic dynamics underlying the human alpha-rhythm electroencephalogram. Biological Cybernetics, 62(1), 55-62. doi:10.1007/BF00217660.

[10] Stam, C. J., Pijn, J. P. M., Suffczynski, P., \& Lopes Da Silva, F. H. (1999). Dynamics of the human alpha rhythm: Evidence for non-linearity? Clinical Neurophysiology, 110(10), 1801-1813. doi:10.1016/S1388-2457(99)00099-1.

[11] Jing, H., \& Takigawa, M. (2000). Topographic analysis of dimension estimates of EEG and filtered rhythms in epileptic patients with complex partial seizures. Biological Cybernetics, 83(5), 391-397. doi:10.1007/s004220000183.

[12] Andrzejak, R. G., Widman, G., Lehnertz, K., Rieke, C., David, P., \& Elger, C. E. (2001). The epileptic process as nonlinear deterministic dynamics in a stochastic environment: An evaluation on mesial temporal lobe epilepsy. Epilepsy Research, 44(23), 129-140. doi:10.1016/S0920-1211(01)00195-4.

[13] Iasemidis, L. D., Pardalos, P., Sackellares, J. C., \& Shiau, D. S. (2001). Quadratic Binary Programming and Dynamical System Approach to Determine the Predictability of Epileptic Seizures. Journal of Combinatorial Optimization, 5(1), 9-26. doi:10.1023/A:1009877331765.

[14] Theiler, J. (1994). On the evidence for low-dimensional chaos in an epileptic electroencephalogram. Physics Letters A, 196(12), 335-341. doi:10.1016/0375-9601(94)91096-0.

[15] Babloyantz, A., \& Destexhe, A. (1986). Low-dimensional chaos in an instance of epilepsy. Proceedings of the National Academy of Sciences of the United States of America, 83(10), 3513-3517. doi:10.1073/pnas.83.10.3513.

[16] Mormann, F., Lehnertz, K., David, P., \& E. Elger, C. (2000). Mean phase coherence as a measure for phase synchronization and its application to the EEG of epilepsy patients. Physica D: Nonlinear Phenomena, 144(3), 358-369. doi:10.1016/S01672789(00)00087-7.

[17] Frank, G. W., Lookman, T., Nerenberg, M. A. H., Essex, C., Lemieux, J., \& Blume, W. (1990). Chaotic time series analyses of epileptic seizures. Physica D: Nonlinear Phenomena, 46(3), 427-438. doi:10.1016/0167-2789(90)90103-V.

[18] Lehnertz, K., \& Elger, C. E. (1998). Can epileptic seizures be predicted? Evidence from nonlinear time series analysis of brain electrical activity. Physical Review Letters, 80(22), 5019-5022. doi:10.1103/PhysRevLett.80.5019.

[19] Le Van Quyen, M., Martinerie, J., Navarro, V., Boon, P., D'Havé, M., Adam, C., ... \& Baulac, M. (2001). Anticipation of epileptic seizures from standard EEG recordings. The Lancet, 357(9251), 183-188. https://doi.org/10.1016/S0140-6736(00)03591-1.

[20] Martinerie, J., Adam, C., Le Van Quyen, M., Baulac, M., Clemenceau, S., Renault, B., \& Varela, F. J. (1998). Epileptic seizures can be anticipated by non-linear analysis. Nature Medicine, 4(10), 1173-1176. doi:10.1038/2667.

[21] Lehnertz, K., Arnhold, J., Grassberger, P., \& Elger, C. E. (2000). Chaos in Brain? In Proceedings of the Workshop on chaos in Brain, World Scientific. 
[22] Iasemidis, L. D., Chris Sackellares, J., Zaveri, H. P., \& Williams, W. J. (1990). Phase space topography and the Lyapunov exponent of electrocorticograms in partial seizures. Brain Topography, 2(3), 187-201. doi:10.1007/BF01140588.

[23] Theiler, J., \& Rapp, P. E. (1996). Re-examination of the evidence for low-dimensional, nonlinear structure in the human electroencephalogram. Electroencephalography and Clinical Neurophysiology, 98(3), 213-222. doi:10.1016/00134694(95)00240-5.

[24] Stam, C. J., Van Woerkom, T. C. A. M., \& Pritchard, W. S. (1996). Use of non-linear EEF measures to characterize EEG changes during mental activity. Electroencephalography and Clinical Neurophysiology, 99(3), 214-224. doi:10.1016/00134694(96)95638-2.

[25] Rapp, P. E., Bashore, T. R., Martinerie, J. M., Albano, A. M., Zimmerman, I. D., \& Mees, A. I. (1989). Dynamics of brain electrical activity. Brain Topography, 2(1-2), 99-118. doi:10.1007/BF01128848.

[26] Jelles, B., Van Birgelen, J. H., Slaets, J. P. J., Hekster, R. E. M., Jonkman, E. J., \& Stam, C. J. (1999). Decrease of non-linear structure in the EEG of Alzheimer patients compared to healthy controls. Clinical Neurophysiology, 110(7), $1159-1167$. doi:10.1016/S1388-2457(99)00013-9.

[27] Stam, C. J., Van Woerkom, T. C. A. M., \& Keunen, R. W. M. (1997). Non-linear analysis of the electroencephalogram in Creutzfeldt-Jakob disease. Biological Cybernetics, 77(4), 247-256. doi:10.1007/s004220050385.

[28] Nandrino, J. L., Pezard, L., Martinerie, J., El Massioui, F., Renault, B., Jouvent, R., Allilaire, J. F., \& Widlöcher, D. (1994). Decrease of complexity in EEG as a symptom of depression. NeuroReport, 5(4), 528-530. doi:10.1097/00001756-19940112000042.

[29] Pijn, J. P. M., Veils, D. N., Van Der Heyden, M. J., DeGoede, J., Van Veelen, C. W. M., \& Lopes Da Silva, F. H. (1997). Nonlinear dynamics of epileptic seizures on basis of intracranial EEG recordings. Brain Topography, 9(4), $249-270$. doi:10.1007/BF01464480.

[30] Mormann, F., Lehnertz, K., David, P., \& E. Elger, C. (2000). Mean phase coherence as a measure for phase synchronization and its application to the EEG of epilepsy patients. Physica D: Nonlinear Phenomena, 144(3), 358-369. doi:10.1016/S01672789(00)00087-7.

[31] Gautama, T., Mandic, D. P., \& Van Hulle, M. M. (2003). Indications of nonlinear structures in brain electrical activity. Physical Review E - Statistical Physics, Plasmas, Fluids, and Related Interdisciplinary Topics, 67(4), 5. doi:10.1103/PhysRevE.67.046204.

[32] Nigam, V. P., \& Graupe, D. (2004). A neural-network-based detection of epilepsy. Neurological Research, 26(1), 55-60. doi:10.1179/016164104773026534.

[33] Güler, I., \& Übeyli, E. D. (2005). Adaptive neuro-fuzzy inference system for classification of EEG signals using wavelet coefficients. Journal of Neuroscience Methods, 148(2), 113-121. doi:10.1016/j.jneumeth.2005.04.013.

[34] Subasi, A. (2007). EEG signal classification using wavelet feature extraction and a mixture of expert model. Expert Systems with Applications, 32(4), 1084-1093. doi:10.1016/j.eswa.2006.02.005.

[35] Adeli, H., Ghosh-Dastidar, S., \& Dadmehr, N. (2007). A wavelet-chaos methodology for analysis of EEGs and EEG subbands to detect seizure and epilepsy. IEEE Transactions on Biomedical Engineering, 54(2), $205-211$. doi:10.1109/TBME.2006.886855.

[36] Guo, L., Rivero, D., Dorado, J., Rabuñal, J. R., \& Pazos, A. (2010). Automatic epileptic seizure detection in EEGs based on line length feature and artificial neural networks. Journal of Neuroscience Methods, 191(1), 101-109. doi:10.1016/j.jneumeth.2010.05.020.

[37] Srinivasan, V., Eswaran, C., \& Sriraam, A. N. (2005). Artificial neural network based epileptic detection using time-domain and frequency-domain features. Journal of Medical Systems, 29(6), 647-660. doi:10.1007/s10916-005-6133-1.

[38] Harikrishnan, K. P., Misra, R., Ambika, G., \& Kembhavi, A. K. (2006). A non-subjective approach to the GP algorithm for analysing noisy time series. Physica D: Nonlinear Phenomena, 215(2), 137-145. doi:10.1016/j.physd.2006.01.027.

[39] Kannathal, N., Acharya, U. R., Lim, C. M., \& Sadasivan, P. K. (2005). Characterization of EEG - A comparative study. Computer Methods and Programs in Biomedicine, 80(1), 17-23. doi:10.1016/j.cmpb.2005.06.005.

[40] Kannathal, N., Choo, M. L., Acharya, U. R., \& Sadasivan, P. K. (2005). Entropies for detection of epilepsy in EEG. Computer Methods and Programs in Biomedicine, 80(3), 187-194. doi:10.1016/j.cmpb.2005.06.012.

[41] Srinivasan, V., Eswaran, C., \& Sriraam, N. (2007). Approximate entropy-based epileptic EEG detection using artificial neural networks. IEEE Transactions on Information Technology in Biomedicine, 11(3), 288-295. doi:10.1109/TITB.2006.884369. 
[42] Nicolaou, N., \& Georgiou, J. (2012). Detection of epileptic electroencephalogram based on Permutation Entropy and Support Vector Machines. Expert Systems with Applications, 39(1), 202-209. doi:10.1016/j.eswa.2011.07.008.

[43] Übeyli, E. D., \& Güler, I. (2007). Features extracted by eigenvector methods for detecting variability of EEG signals. Pattern Recognition Letters, 28(5), 592-603. doi:10.1016/j.patrec.2006.10.004.

[44] Polat, K., \& Güneş, S. (2008). Artificial immune recognition system with fuzzy resource allocation mechanism classifier, principal component analysis and FFT method based new hybrid automated identification system for classification of EEG signals. Expert Systems with Applications, 34(3), 2039-2048. doi:10.1016/j.eswa.2007.02.009.

[45] Kumar, Y., Dewal, M. L., \& Anand, R. S. (2014). Epileptic seizures detection in EEG using DWT-based ApEn and artificial neural network. Signal, Image and Video Processing, 8(7), 1323-1334. doi:10.1007/s11760-012-0362-9.

[46] Subasi, A., \& Gursoy, M. I. (2010). EEG signal classification using PCA, ICA, LDA and support vector machines. Expert Systems with Applications, 37(12), 8659-8666. doi:10.1016/j.eswa.2010.06.065.

[47] Orhan, U., Hekim, M., \& Ozer, M. (2011). EEG signals classification using the K-means clustering and a multilayer perceptron neural network model. Expert Systems with Applications, 38(10), 13475-13481. doi:10.1016/j.eswa.2011.04.149.

[48] Wang, L., Xue, W., Li, Y., Luo, M., Huang, J., Cui, W., \& Huang, C. (2017). Automatic epileptic seizure detection in EEG signals using multi-domain feature extraction and nonlinear analysis. Entropy, 19(6). doi:10.3390/e19060222.

[49] Gajic, D., Djurovic, Z., Gligorijevic, J., Di Gennaro, S., \& Savic-Gajic, I. (2015). Detection of epileptiform activity in EEG signals based on time-frequency and non-linear analysis. Frontiers in Computational Neuroscience, 9(MAR). doi:10.3389/fncom.2015.00038.

[50] Wang, D., Miao, D., \& Xie, C. (2011). Best basis-based wavelet packet entropy feature extraction and hierarchical EEG classification for epileptic detection. Expert Systems with Applications, 38(11), 14314-14320. doi:10.1016/j.eswa.2011.05.096.

[51] Fergus, P., Hignett, D., Hussain, A., Al-Jumeily, D., \& Abdel-Aziz, K. (2015). Automatic epileptic seizure detection using scalp EEG and advanced artificial intelligence techniques. BioMed Research International, 2015. doi:10.1155/2015/986736.

[52] Hsu, K. C., \& Yu, S. N. (2010). Detection of seizures in EEG using subband nonlinear parameters and genetic algorithm. Computers in Biology and Medicine, 40(10), 823-830. doi:10.1016/j.compbiomed.2010.08.005.

[53] Sharmila, A., Madan, S., \& Srivastava, K. (2018). Epilepsy detection using dwt based hurst exponent and SVM, K-NN classifiers. Serbian Journal of Experimental and Clinical Research, 19(4), 311-319. doi:10.1515/SJECR-2017-0043.

[54] Alickovic, E., Kevric, J., \& Subasi, A. (2018). Performance evaluation of empirical mode decomposition, discrete wavelet transform, and wavelet packed decomposition for automated epileptic seizure detection and prediction. In Biomedical Signal Processing and Control (Vol. 39, pp. 94-102). doi:10.1016/j.bspc.2017.07.022.

[55] Satapathy, S. K., Dehuri, S., \& Jagadev, A. K. (2016). An empirical analysis of different machine learning techniques for classification of EEG signal to detect epileptic seizure. International Journal of Applied Engineering Research, 11(1), 120-129.

[56] Subasi, A., Kevric, J., \& Abdullah Canbaz, M. (2019). Epileptic seizure detection using hybrid machine learning methods. Neural Computing and Applications, 31(1), 317-325. doi:10.1007/s00521-017-3003-y.

[57] Hussain, L. (2018). Detecting epileptic seizure with different feature extracting strategies using robust machine learning classification techniques by applying advance parameter optimization approach. Cognitive Neurodynamics, 12(3), $271-294$. doi:10.1007/s11571-018-9477-1.

[58] Rosas-Romero, R., Guevara, E., Peng, K., Nguyen, D. K., Lesage, F., Pouliot, P., \& Lima-Saad, W. E. (2019). Prediction of epileptic seizures with convolutional neural networks and functional near-infrared spectroscopy signals. Computers in Biology and Medicine, 111(103355). doi:10.1016/j.compbiomed.2019.103355.

[59] Gusnanto, A., Calza, S., \& Pawitan, Y. (2007). Identification of differentially expressed genes and false discovery rate in microarray studies. Current Opinion in Lipidology, 18(2), 187-193. doi:10.1097/MOL.0b013e3280895d6f.

[60] Dudoit, S., Yang, Y. H., Callow, M. J., \& Speed, T. P. (2002). Statistical methods for identifying differentially expressed genes in replicated cDNA microarray experiments. Statistica sinica, 111-139.

[61] Pepe MS. (2003). The statistical evaluation of medical tests for classification and prediction. Oxford (UK): Oxford University Press; ISBN: 9780198565826.

[62] Parodi, S., Muselli, M., Fontana, V., \& Bonassi, S. (2003). ROC curves are a suitable and flexible tool for the analysis of gene expression profiles. Cytogenetic and Genome Research, 101(1), 90-91. doi:10.1159/000074404.

[63] Patidar, S., \& Panigrahi, T. (2017). Detection of epileptic seizure using Kraskov entropy applied on tunable-Q wavelet transform of EEG signals. Biomedical Signal Processing and Control, 34, 74-80. doi:10.1016/j.bspc.2017.01.001.

[64] Acharya, U. R., Oh, S. L., Hagiwara, Y., Tan, J. H., \& Adeli, H. (2018). Deep convolutional neural network for the automated detection and diagnosis of seizure using EEG signals. Computers in Biology and Medicine, 100, $270-278$. doi:10.1016/j.compbiomed.2017.09.017. 
[65] Zhou, M., Tian, C., Cao, R., Wang, B., Niu, Y., Hu, T., Guo, H., \& Xiang, J. (2018). Epileptic seizure detection based on EEG signals and CNN. Frontiers in Neuroinformatics, 12, 95. doi:10.3389/fninf.2018.00095.

[66] Tsipouras, M. G. (2019). Spectral information of EEG signals with respect to epilepsy classification. Eurasip Journal on Advances in Signal Processing, 2019(1). doi:10.1186/s13634-019-0606-8.

[67] Bayrak, S., Yucel, E., Takci, H., \& Samli, R. (2021). Classification of epileptic electroencephalograms using time-frequency and back propagation methods. Computers, Materials and Continua, 69(2), 1427-1446. doi:10.32604/cmc.2021.015524.

[68] Wang, Z., Wang, Q., Liu, J., Liang, Z., \& Xu, J. (2020). New SAR imaging algorithm via the optimal time-frequency transform domain. Computers, Materials and Continua. https://doi.org/10.32604/cmc.2020.011909

[69] Sharma, R., Sircar, P., \& Pachori, R. B. (2020). Automated Seizure Classification Using Deep Neural Network Based on Autoencoder. In Handbook of Research on Advancements of Artificial Intelligence in Healthcare Engineering, IGI Global Publications 2020 (pp. 1-19). doi:10.4018/978-1-7998-2120-5.ch001.

[70] Abdelhameed, A., \& Bayoumi, M. (2021). A Deep Learning Approach for Automatic Seizure Detection in Children with Epilepsy. Frontiers in Computational Neuroscience, 15. doi:10.3389/fncom.2021.650050. 